\title{
EL PIXOY COMO MATERIAL DE CONSERVACIÓN DE PINTURA MURAL Y RELIEVES POLICROMOS EN EL ÁREA MAYA
}

\author{
Ma. Cristina Ruiz Martín \\ Centro INAH Yucatán
}

\begin{abstract}
Resumen: Ante la problemática que se ha enfrentado en sitios arqueológicos del área maya en la conservación de pintura mural y relieves en estuco policromos que se preservan in situ. se ha buscado en nuevos materiales contrarrestar efectos de deterioro que pueden generar la pérdida de estos bienes. Dentro de los productos utilizados están los extractos vegetales. empleados principalmente como aditivos a morteros de cal y en el caso de Ek 'Balam, como consolidante de capas de color. Se ha desarrollado una investigación para evaluar uno de estos materiales, el pixoy (Guazuma ulmifolia), y determinar su efectividad en la conservación arqueológica.
\end{abstract}

Palabras a.AvE: conservación arqueológica, pintura mural, relieves en estuco policromos, fijativo, goma de pixoy.

\begin{abstract}
Anstract: Because of the conservation problems of the mural paintings and color stucco reliefs from the archaeological sites of the Mayan area which are preserved in situ, the investigation of new materials that resist deterioration effects have been a very important goal for restorers. One of these products are the vegetables extracts, used like additives for the lime, and in the case of Ek 'Balam as fixatives of pictorial layer. This investigation has been developed to evaluate one of these materials, pixoy gum (Guazuma ulmifolia), and to determine its effectiveness in the archaeological conservation.
\end{abstract}

Kfyworns: archaeological conservation, mural paintings, color stucco reliefs, fixative, pixoy gum.

REcEPCoÓn: 28 de octubre de 2007.

Acr.PTACION: 23 de febrero de 2009 . 



\title{
EL PIXOY COMO MATERIAL DE CONSERVACIÓN DE PINTURA MURAL Y RELIEVES POLICROMOS EN EL ÁREA MAYA
}

\author{
Ma. Cristina Ruiz Martín \\ Centro INAH Yucatán
}

\section{Introducción}

Uno de los retos más importantes que ha tenido la restauración es la conservación de los bienes arqueológicos inmuebles por destino, pintura mural, relieves en estuco y relieves en piedra. Una serie de factores han incidido en que bienes que lograron conservarse por varios siglos, desde que fueron construidos hasta su época de enterramiento, hayan sufrido un deterioro acelerado a tan sólo unos años de haber sido descubiertos y liberados.

Las características del contexto ambiental, el estado de conservación del edificio en el que se localizan, la aplicación de procesos y de materiales no efectivos y una falta de visión general de la conservación como algo que corresponde a todos los que se relacionan con el patrimonio arqueológico y no sólo de los restauradores, son las principales causas de que este tipo de bienes se encuentren en las condiciones actuales.

Puntualizando en el caso de los sitios arqueológicos del área maya la problemática es seria, en innumerables casos ha sido imposible, por su complejidad y variedad, controlar por completo los factores de deterioro a pesar de entenderlos, asimismo no se han encontrado materiales de restauración que cumplan de manera efectiva en la solución de algunos efectos de deterioro, como es el caso particular de la consolidación y fijado de capas pictóricas disgregadas.

Sin embargo, $\mathrm{y}$ a pesar de la problemática mencionada, durante el desarrollo y consolidación de la disciplina de la restauración se ha conseguido un avance fundamental en el estudio y comprensión de los mecanismos de deterioro. Por su parte, se han logrado conformar grandes bases de datos que nos informan sobre las características técnicas y materiales de los bienes arqueológicos inmuebles por destino. Ambos avances han sido de gran valor para conocer la forma en que estos bienes se comportan de manera intrínseca y cómo interactúan con el edificio y su medio, esto ha permitido comprender mejor el porqué de su estado actual, más no ha dado soluciones contundentes para su conservación.

Una parte clave para entender por qué, a pesar de los trabajos de conservación hechos con materiales y procesos adecuados y efectivos en diversos sitios arqueológicos, los bienes continúan deteriorándose, es ubicarlos dentro de su 
muy particular y al mismo tiempo complejo contexto. Es fundamental considerar que los sitios del área maya, por su ubicación geográfica, están expuestos a altos porcentajes de humedad relativa, lluvias, elevadas temperaturas, desarrollo de microorganismos, plantas superiores y animales, principales factores de deterioro de los bienes que en ellos se conservan. Asimismo éstos pertenecen a un edificio con un estado de conservación determinado, el cual influirá de manera directa en las condiciones en las que estarán los elementos decorativos que sustenta.

Ambos factores, tanto las características climáticas, de flora y fauna, así como el edificio, no permanecen estables, se mantienen en constante cambio. Por lo tanto, a pesar de que se logren contrarrestar otros factores de alteración a partir de una intervención de restauración, los bienes inmuebles por destino no lograrán su conservación si antes no se estabiliza la estructura en la que se ubican y sin haber logrado un control de la incidencia de las condiciones climáticas en ellos, hechos que hasta el momento no ha sido posible alcanzar.

Ante esta situación la restauración ha procurado que a partir de acciones de conservación preventiva como colocación de techos, construcción de drenes, uso de recubrimientos de protección, re-enterramientos, entre otros; junto con las intervenciones directas de restauración se logre en la mayor medida y por el mayor tiempo posible la conservación de los bienes.

Breve reseña histórica del uso de productos fijativos en sitios arqueológicos

En cuanto a los procesos de conservación directa el camino no ha sido del todo positivo, se han cometido errores debido al desconocimiento de los bienes en sí, de su proceso de deterioro y de los productos de conservación. Este problema se generó principalmente en el periodo comprendido entre las décadas de los sesenta y los ochenta, por el uso de polímeros sintéticos, los cuales aparentaban gran efectividad y durabilidad, hasta que a finales de los ochenta comenzaron a hacer evidentes los graves problemas generados por el uso de estos productos, que en algunos casos llegaron a ocasionar la pérdida de pinturas murales y relieves policromos.

Este tema fue analizado y estudiado por el restaurador Luciano Cedillo en su tesis de licenciatura La Conservación de zonas arqueológicas: tres décadas de trabajo, en donde evaluó el comportamiento y los efectos del uso de polímeros sintéticos en sitios arqueológicos de distintas zonas del país, entre ellas: Palenque, Kohunlich, Comalcalco y Teotihuacán (Cedillo, 1991).

A partir de esa investigación y de la experiencia de restauradores profesionales en sitios como Balamkú y Ek'Balam, por mencionar algunos, se concluyó de forma determinante que los polímeros sintéticos, más allá de favorecer la conservación de los elementos en estuco y piedra, se convirtieron en importantes causas de deterioro. 
La inestabilidad de los productos sintéticos empleados como fijativos, ante condiciones climáticas con elevadas temperaturas, alto porcentaje de humedad relativa, exposición al sol y a la precipitación pluvial directa, produjo la formación de películas plásticas rígidas y quebradizas sobre el sustrato pictórico original. "Estos efectos de deterioro son cambios químicos del polímero (entrecruzamiento entre las cadenas poliméricas, rompimiento de las cadenas o la oxidación del polímero), ocasionados por su exposición a la luz, a altas temperaturas, oxígeno y contaminantes" (Horie, 1997: 31-32). Al respecto Martínez de las Marías (1972) considera:

Por acción de agentes físicos o químicos puede producirse la rotura total o parcial de las cadenas macromoleculares de los altos polímeros, con la consiguiente disminución del grado de polimerización. Entre los agentes físicos que pueden producir una despolimerización citaremos [...] el calor, la luz visible y UV, los ultrasonidos y las radiaciones energéticas. Estos agentes producen una degradación más o menos extensa, que es en función de la intensidad y tiempo de acción y de la estabilidad de los materiales $[\ldots .$. , es decir, de la estructura y constitución del producto molecular (Martínez de las Marías, 1972: 190).

El proceso de deterioro sufrido por los polímeros hizo que éstos, además de perder su efectividad y dejar de cumplir su función, incidieran directamente en la formación de escamas y pulverulencia de estratos pictóricos, disgregación de estuco y aplanados, alteración cromática de capas pictóricas, sellado e impermeabilización de superficies con la consecuente disolución y cristalización de sales al interior, entre otros.

Una vez identificados los efectos de los productos sintéticos, se inició una nueva etapa en la conservación de bienes arqueológicos inmuebles, en la cual el uso de materiales de naturaleza igual o similar a los empleados en la manufactura de los originales fue la tendencia; la cal y sus derivados resultaron ser la base para la preparación de materiales de restauración: en combinación con cargas, morteros para reintegraciones formales y volumétricas (resanes y ribetes); muy bien colada y diluida, lechada para consolidaciones de material interno disgregado; y finalmente utilizando sólo el agua obtenida del apagado o la hidratación de la cal, como fijativo de capas pictóricas disgregadas y también consolidante.

Los resultados en la práctica del uso de la cal como producto de restauración ha sido positiva, particularmente usada en morteros y como consolidante, sin embargo no ha logrado contrarrestar por completo algunos efectos, en particular los relacionados con el fijado de pinturas deterioradas, siendo en algunas ocasiones un paliativo y en otras efectiva pero causante de alteraciones en la apreciación cromática de capas pictóricas al producir un velo blanquecino en superficie. Además cabe señalar que el agua de cal presenta algunos inconvenientes, como lo son la falta de penetración y la lenta reacción que se da entre el $\mathrm{CaOH}$ (hidróxido de calcio) y el $\mathrm{CO}_{2}$ (bióxido de carbono) debido a la baja solubilidad de este último en el agua Además, se requiere gran cantidad de agua, mucho 
tiempo y constancia para hacer que el proceso sea realmente efectivo (Brajer y Kalsbeek, 1999: 152-154).

A partir de la década de los noventa se empezó a experimentar con el uso de extractos vegetales como aditivos a los morteros de cal, siguiendo una tradición conocida a través de las escasas fuentes que la mencionan Relación de las cosas de Yucatán de fray Diego de Landa (1986), El Templo de los Guerreros de Earl H. Morris (1931) (Jáidar, 2006: 92-93) y People of the Serpent. Life and Adventure Among the Mayas de Edward Thompson (1965). Sin embargo, y como apoyo a las menciones que se hacen en las fuentes, a partir de análisis con técnicas instrumentales ha sido posible identificar estos materiales en la composición de los estucos y aplanados.'

Del uso como aditivo en morteros, se derivó su empleo como fijativo de capas pictóricas, al observar las características glutinosas y adhesivas de los extractos vegetales obtenidos del macerado de corteza de árboles, se consideró viable el que fueran capaces de devolverle a una estrato de color pulverulento su cohesión y adhesión; siendo las gomas del chucúm (Acacia albicans) en Chacmultún, Yucatán y en Becán, Campeche y el pixoy (Guazuma ulmifolia) en Ek'Balam, Yucatán, las que se han empleado principalmente.

\section{El uso del extracto de pixoy como fijativo y su evaluación}

A partir de la experiencia obtenida al utilizar el extracto de pixoy como fijativo y consolidante en el sitio arqueológico de Ek Balam, Yucatán, se decidió realizar una evaluación que permitiera saber si la goma de pixoy estaba siendo realmente efectiva en el control de procesos de disgregación pictórica.

La investigación, más allá de tener un objetivo puramente práctico, se consideró de gran importancia porque estos productos se han utilizado desde hace varios años sin saber su verdadero comportamiento al interactuar con un estrato disgregado en los contextos climáticos del área maya. Considerando la experiencia negativa obtenida del uso de polímeros sintéticos sin una evaluación previa de su reacción ante las condiciones de los sitios arqueológicos, resultaba fundamental evaluar estos "nuevos materiales", ya que a pesar de que por su propia naturaleza distan mucho de tener un comportamiento similar a los sintéticos, es indudable que se estaba cayendo en el mismo error que con los polímeros al emplearlos de forma desmedida y sin haberlos estudiado a profundidad, bajo la justificación de que por ser "naturales" y estar identificados en la manufactura original de los estratos pictóricos de pinturas murales y relieves en estuco, no

\footnotetext{
${ }^{1}$ Véase: Littman (1960 y 1965), Diana Magaloni (1997, 2000, 2001), Vázquez del Mercado y Villegas Yduñate (1996), Vázquez Negrete y Velásquez Castillo (1994), Mateos González (1997), Vázquez de Ágredos Pascual (2004 y 2006). Alonso Olvera, et al. (2005).
} 
podría tener efectos nocivos en dichos bienes, y tal vez esto es real, sin embargo, nada de lo anterior podía asegurar su efectividad aunque a un nivel macroscópico los resultados así lo indicaran.

Debido a que en la bibliografía consultada no se encontró una metodología ya establecida para evaluar un producto fijativo, fue necesario proponer y probar un nuevo sistema. Para la construcción de la metodología y del diseño experimental con el que se evaluó la efectividad del producto se siguieron los siguientes pasos:

1. Se definió qué es un proceso de fijado y cuáles son las características de un producto fijativo ideal. Esto permitió determinar las características a considerar al momento de diseñar las pruebas de evaluación.

2. Se caracterizó la naturaleza del extracto de pixoy. Al conocer las propiedades del producto fue posible comprender los resultados obtenidos en la evaluación, ya que muchos de ellos fueron determinados por la naturaleza del pixoy.

3. Se estableció una serie de pruebas que permitieron calificar cada una de las propiedades con las que debe contar el pixoy para ser un fijativo efectivo. Propiedades mecánicas, propiedades ópticas y solubilidad.

4. Se llevó a la práctica el diseño experimental en muestras de pintura mural original y muestras problema.

\section{El proceso de fijado}

y las características de un fijativo ideal

La función del proceso de fijado es devolver a una capa pulverulenta cohesión entre las partículas de color y su adhesión al soporte. Para este proceso se utiliza un producto denominado fijativo, el cual debe contar con propiedades específicas para que sea funcional y efectivo. Una de las principales características es el hecho de que se aplica en estado líquido, con el objetivo de que impregne y penetre el estrato pulverulento, pero posteriormente debe adquirir un estado sólido, ya que es lo que va a producir la capa que proveerá la cohesión y adhesión necesaria.

De manera muy general, es necesario considerar diversas propiedades del producto tales como las características físico-químicas del consolidante y del disolvente que sirve de vehículo, la viscosidad de la mezcla y la porosidad del sustrato donde se aplica. Una vez seco es necesario que la capa formada sea flexible, resistente a los agentes atmosféricos y biológicos, debe ser transparente y no debe modificar el brillo o la opacidad del original y por supuesto deberá conseguir el nivel de penetración/impregnación y consolidación indispensable para contrarrestar el efecto de pulverulencia. 


\section{El extracto de pixoy}

El árbol

Pixoy es el nombre en maya del árbol denominado como Guazuma ulmifolia. ${ }^{2}$ Pertenece a la familia de dicotiledóneas Esterculiáceas o Sterculiacea, presenta flor y fruto (Standley, 1977: 423).

La forma más sencilla de identificar este árbol es por su fruto que, de acuerdo con la descripción de Roys es una cápsula negra, de textura similar a la madera (Roys, 1931: 276) con pequeñas protuberancias en superficie. El color oscuro lo adquiere cuando está completamente maduro, lo cual sucede casi todo el año, permanecen durante largo tiempo en el árbol (Pennington, 1998: 382). (Imagen 1)

\section{Características del extracto de pixoy}

A partir de observaciones macroscópicas, se describe el extracto del pixoy como un material soluble en agua potable, lo cual indica que se trata de un polisacárido. La solución es de color rojo-naranja intenso, transparente, densa, viscosa y de características glutinosas. El líquido no es completamente homogéneo, después de mantenerlo en reposo se observan partículas asentadas en el fondo del recipiente. El extracto va perdiendo su transparencia con el paso del tiempo, además se intensifica el color naranja y disminuye el rojizo tornándose más bien en una mezcla de color café-naranja, mismo color que toma el producto al secar sobre un portaobjetos. Si se mantiene el extracto en refrigeración y en una botella bien cerrada conserva sus propiedades hasta un año después de su obtención, sin embargo, en las condiciones de campo éste comienza a pudrirse a la semana de haber sido extraído, generando un aroma a fermentado (Ruiz, 2007: 106).

En cuanto a la constitución química del extracto se corroboró que se trata de un polisacárido que debe su coloración rojiza a un alto porcentaje de taninos en su composición. También se detectó la presencia de material inorgánico: calcio y cloro, debido probablemente, el primero, al suelo de caliza de la región y el segundo al uso de agua potable en la preparación del extracto analizado. Los resultados de la caracterización se obtuvieron a partir de análisis con técnicas analíticas empleando Espectroscopia de Infrarrojo con Transformada de Fourier (FTIR) y Microscopio Electrónico de Barrido con Microsonda de Electrones (MEB/ EDS), así como sencillas pruebas a la gota (Ruiz, 2007: 113).

\footnotetext{
${ }^{2}$ De acuerdo con cada región, el árbol recibe diferentes nombres: Guácima, guácimo (nombres más comúnmente usados en toda su área de distribución); cuaulote (del náhuatl cuauolotl, Gro., Oax., Chis.); aquiche, kabal-pixoy, pixoy (maya, Yuc.); acashti (totonaco, Ver.): ajillá (guarijío, Son., Sin.): majagua de toro (Oax.); nocuana-yana (zapoteco, Oax.); palote negro (Mich.); parandesicua (tarasco, Mich.); tablote, tzuni, tzuyui (Chis.), uiguie (popoluca, Ver.); ya-ana (zapoteco, Oax.); yaco granadillo, yaco de venado (Oax.) zam-mi (chontal, Oax.); áquich (huasteco, S.L.P); ajya (mayo, Son.); $k^{\prime}$ olin kakaw (tzetzal, Chis.) (Standley, 1977: 423).
} 


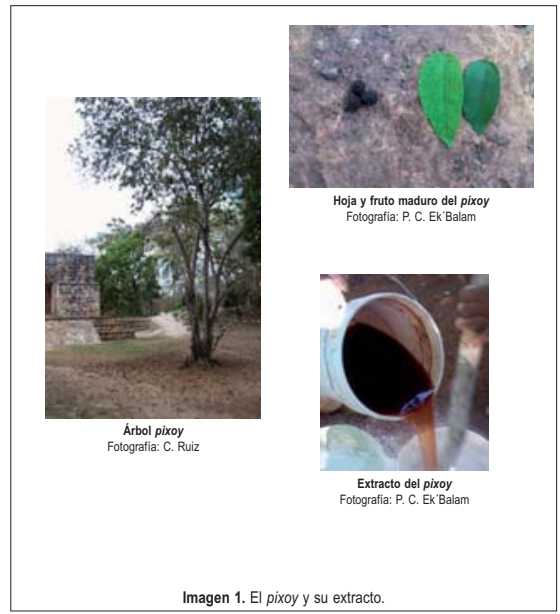

Es muy importante señalar que la caracterización de la naturaleza del extracto del pixoy fue fundamental para comprender los resultados obtenidos al evaluarlo como fijativo, como se explicará más adelante.

\section{Metodología de evaluación}

Para evaluar el extracto de pixoy los factores a considerar se definieron a partir de las características de un fijativo ideal, se clasificaron en tres grupos: propiedades ópticas, en las que se incluyeron el color, la transparencia y el brillo; propiedades mecánicas: distribución y comportamiento en superficie, penetración e 
impregnación, resistencia a la abrasión y adhesión de los pigmentos al soporte; y finalmente se hicieron pruebas de solubilidad empleando tanto solventes polares como no polares, esto una vez que habían pasado varios meses de haberse aplicado los fijativos en las muestras.

Para la extracción de la goma del pixoy primero se obtienen fragmentos de la corteza, de $1 \mathrm{~cm}$ de espesor aproximadamente y $20-30 \mathrm{~cm}$ de largo, posteriormente se golpean con una piedra o un pedazo de madera para abrir y ablandar las fibras y así facilitar la extracción de la goma, se coloca en cubetas de agua (en este caso se hizo tanto en agua potable como de cal) y se deja macerando por dos o tres días. Posteriormente se cuela la mezcla para limpiarla y separar la corteza del líquido. (Imagen 2)

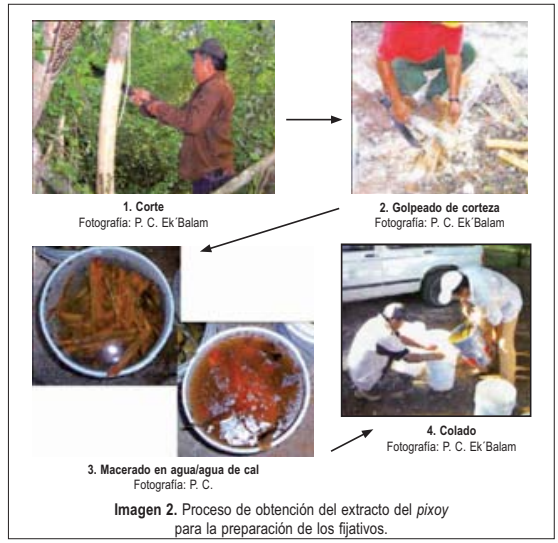




\section{Evaluación de los fijativos preparados con goma de pixoy}

Para la evaluación se prepararon tres fijativos distintos, tomando en cuenta las diversas formas en que se habían utilizado los productos en el trabajo en campo. El primer fijativo se obtuvo al macerar la corteza de pixoy en agua de cal, el segundo macerando la corteza en agua potable y mezclando el extracto en proporción 1:1 con agua de cal y finalmente en el tercer fijativo sólo se utilizó el extracto adquirido de macerar la corteza en agua potable.

Cada uno de estos fijativos presentó características diferentes desde que fueron preparados, se describen a continuación:

- Fijativo 1 Pixoy macerado en agua de cal. El extracto es ligeramente menos viscoso pero más denso que el preparado en agua; el color rojo es mucho más intenso y oscuro y el aroma de la madera no es tan fuerte al mezclarse con el olor de la cal; es menos traslúcido que el extracto obtenido con agua potable.

- Fijativo 2 Pixoy macerado en agua + agua de cal en una proporción 1:1. Recién preparado, el fijativo es de color naranja-café, es espeso y menos traslúcido que el fijativo 3, el aroma es el de la corteza mojada. Es importante mencionar que 10 minutos después de realizar esta mezcla se da una separación de componentes formándose pequeños conjuntos de partículas amorfas de color café oscuro-rojizas que flotan y posteriormente se asientan en el líquido, mismo que toma un color amarillo claro, perdiendo su viscosidad y cualidades adhesivas. Es probable que esta reacción se dé entre la cal y los taninos, ya que los segundos son incompatibles con la alcalinidad del hidróxido de calcio. ${ }^{3}$

- Fijativo 3 Pixoy macerado en agua. Es una mezcla de color naranja rojiza y traslúcida, su aroma es de madera mojada. (Imagen 3)

Los fijativos se aplicaron en tres grupos distintos de muestras, constituidos por 6 fragmentos de pintura mural original (sin problema de pulverulencia) y 3 muestras problema que se hicieron con el objetivo de que la capa pictórica presentara pulverulencia. Esto fue necesario debido a que del material original disponible para los análisis no existieron fragmentos que tuvieran este problema de deterioro. Las muestras originales permitieron evaluar principalmente propiedades ópticas. A cada grupo se agregó un portaobjetos sobre los que también se aplicaron los fijativos, lo que fue fundamental para observar muchas de las características de las capas formadas por los fijativos. (Imagen 4)

\footnotetext{
${ }^{3}$ El ácido tánico del cual están conformados los taninos es incompatible con el agua de cal (Budavari, 1989: 1431).
} 


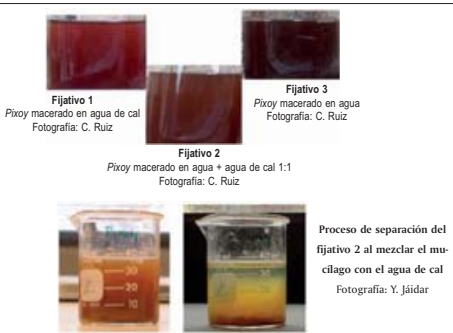

Imagen 3. Fijativos preparados con el extracto del pixoy, se pueden observar las distintas coloraciones y además el particular proceso de separación del fijativo 2.

Tanto las muestras como los portaobjetos se asperjaron con los fijativos, este proceso se hizo de forma constante y sin dejar que secaran por completo, ya que de esta forma se pretendía favorecer la penetración e impregnación de los productos en las muestras. Esto se hizo hasta que se observó a nivel macroscópico que ya no continuaba el efecto de pulverulencia.

La cantidad de fijativo varió en cada caso, del que se aplicó menor cantidad fue del Fijativo 1 Pixoy macerado en agua de cal, mientras que el Fijativo 2 Pixoy macerado en agua potable 1:1 con agua de cal fue el que se asperjó en mayor cantidad, siendo el Fijativo 3 Pixoy macerado en agua potable, el que tuvo un comportamiento intermedio respecto a los otros dos. (Imagen 5)

Pruebas de evaluación y sus resultados

PROPIEDADES ÓPTICAS

$\underline{\text { Color }}$

Desde el momento de la aplicación de los productos fue evidente el radical cambio de coloración que adquirieron las muestras, eso como consecuencia del color de los fijativos. En un principio se consideró que al ser un material orgánico sería posible que la alteración cromática disminuyera considerablemente al 


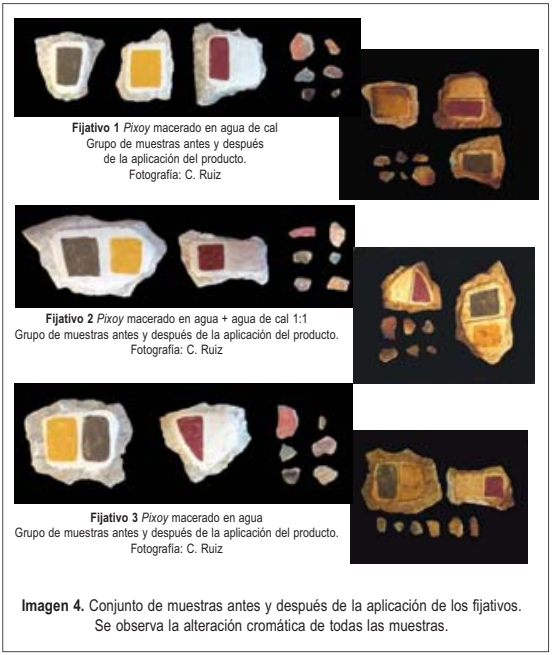

exponer las muestras a la luz del sol, por lo anterior una muestra problema de cada fijativo, con la mitad cubierta por una cartulina negra, se mantuvo expuesta a luz del sol durante 4 meses. Después de este tiempo la coloración naranjarojiza que habían adquirido las muestras no tuvo una disminución considerable. El color no disminuyó debido a que la coloración rojiza de los taninos, que a su vez es la sustancia que da el color a la goma, se debe a la presencia de óxidos de hierro, material inorgánico sumamente estable que conserva su color aun ante la radiación solar. (Tabla 1) 
A. Antes de la aplicación de los fijativos

B. Después de la aplicación de los fijativos

C. Después de cinco meses de exponer al sol sólo una parte de las muestras

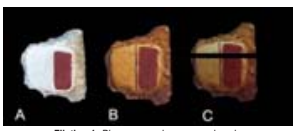

Fijativo 1. Pixoy macerado en agua de cal.

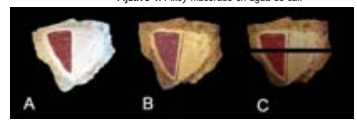

Fijativo 2. Pixoy macerado en agua + agua de cal 1:1.

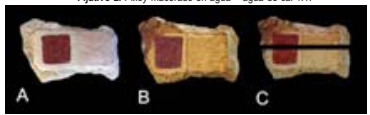

Fijativo 3. Pixoy macerado en agua.

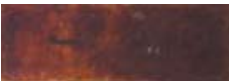

Superficie mate a nivel macro y microscópico.

Fotografia: J. Vàzquez

Fijativo 1. Pixoy macerado en agua de cal Coloración roja-café, opacidad casi absoluta. Fotografia: C. Ruiz

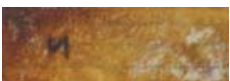

Superficie mate a nivel macro. Brillo muy localizado a nivel microscópico. Fotografia: J. Vázquez

Fijativo 2. Pixoy macerado en agua + agua de cal $1: 1$ Coloración naranja-amarila y menor opacidad respecto a fijativos 1 y 2

Fotografia: C. Ruiz

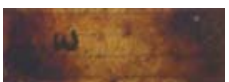

Superficie mate a nivel macro. Mayor brillo a nivel microscópico respecto a fijativos 1 \% 2

Fotografia: J. Vázquez

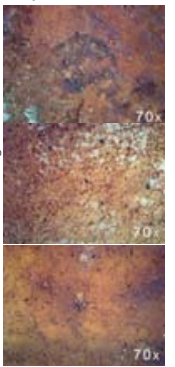

Fijativo 3. Pixoy macerado en agua. Coloración café-amarlla y opacidad media Fotografia: C. Ruiz

Imagen 5. Evaluación del color, transparencia y brillo de los fijativos. 


\begin{tabular}{|l|l|}
\hline & \multicolumn{1}{|c|}{ Observaciones } \\
\hline $\begin{array}{l}\text { Fijativo 1 } \\
\text { Pixoy macerado en agua } \\
\text { de cal }\end{array}$ & $\begin{array}{l}\text { Este producto presentó la coloración más intensa. Es de } \\
\text { color rojizo y tiende al café oscuro. Modifica por completo } \\
\text { las características cromáticas de las muestras. }\end{array}$ \\
\hline $\begin{array}{l}\text { Fijativo 2 } \\
\text { Pixoy macerado en agua + } \\
\text { agua de cal 1:1 }\end{array}$ & $\begin{array}{l}\text { En este caso la coloración fue la de menor intensidad. } \\
\text { Tiende más al naranja y al amarillo oscuro. Modifica las } \\
\text { caracteristicas originales de las capas pictóricas. }\end{array}$ \\
\hline $\begin{array}{l}\text { Fijativo 3 } \\
\text { Pixoy macerado en agua }\end{array}$ & $\begin{array}{l}\text { Comparando con los otros dos fijativos, éste es el que } \\
\text { presenta una coloración intermedia. El color que genera es } \\
\text { café-amarillo. También modifica las características de las } \\
\text { pinturas. }\end{array}$ \\
\hline
\end{tabular}

Tabla 1. Coloración de las capas formadas por los tres fijativos.

\section{$\underline{\text { Transparencia }}$}

Esta propiedad se evaluó principalmente en los portaobjetos que fueron asperjados junto con las muestras, ya que por ser de vidrio permitieron observar las características de las capas formadas por cada fijativo. Ninguno de los productos forma una capa transparente, todas son parcialmente traslúcidas, propiedad que se pierde conforme aumenta la cantidad de fijativo aplicado. (Tabla 2)

\begin{tabular}{|l|l|}
\hline & \multicolumn{1}{|c|}{ Observaciones } \\
\hline $\begin{array}{l}\text { Fijativo 1 } \\
\begin{array}{l}\text { Pixoy macerado en } \\
\text { agua de cal }\end{array}\end{array}$ & $\begin{array}{l}\text { La capa formada por este fijativo es la menos traslúcida; en las } \\
\text { zonas de mayor concentración es casi totalmente opaca. }\end{array}$ \\
\hline $\begin{array}{l}\text { Fijativo 2 } \\
\text { Pixoy macerado en } \\
\text { agua + agua de cal 1:1 }\end{array}$ & $\begin{array}{l}\text { Este fijativo genera la capa más traslúcida de los tres productos. } \\
\text { transparencia. }\end{array}$ \\
\hline $\begin{array}{l}\text { Fijativo 3 } \\
\text { Pixoy macerado en } \\
\text { agua }\end{array}$ & $\begin{array}{l}\text { La capa que se crea al aplicar el fijativo 3 tiene una traslucidez } \\
\text { intermedia entre los fijativos 1 y 2, disminuye conforme aumenta } \\
\text { la concentración del producto. }\end{array}$ \\
\hline
\end{tabular}

Tabla 2. Transparencia de las capas formadas por los tres fijativos.

Brillo

Para evaluar esta característica se hicieron observaciones en todas las muestras a nivel macro y microscópico con ayuda de un microscopio estereoscópico. Ade- 
más se observaron los portaobjetos en un microscopio óptico. En general y a nivel macro las películas formadas por todos los fijativos son mate, sin embargo, en las observaciones microscópicas fue posible observar algunas zonas de brillo, principalmente con los fijativos 2 y 3 . (Tabla 3)

PROPIEDADES ÓPTICAS. ANÁLISIS DE RESULTADOS

La coloración del pixoy fue un factor sustantivo para desaprobar el uso de los productos evaluados, pues se contrapone a una de las primeras características con las que debe contar un fijativo: ser incolora. Además, el color de los productos incide directamente en la falta de transparencia de las capas formadas, característica indispensable para cualquier fijativo, ya que éste no puede ni debe modificar las características pictóricas originales de la pintura o el relieve (Ruiz, 2007: 142-143).

A un nivel macroscópico todas las muestras resultaron mates, observándose sólo zonas con brillo al utilizar lentes de aumento. Esto se debe a la naturaleza de los fijativos. Al tratarse de un polisacárido, las moléculas que lo conforman son de gran tamaño, y al aplicar un producto con esa composición sobre una superficie, su distribución es muy irregular, lo que provoca una reflexión difusa de la luz y por lo tanto un efecto mate (Mora y Mora, 1984: 220; Masschelein, 1995: 17). (Imagen 6)

Propiedades mecánicas

DISTRIBUCIÓN Y COMPORTAMIENTO EN SUPERFICIE

Esta característica se evaluó tanto en las muestras de pintura como en los portaobjetos, se hicieron observaciones a nivel macroscópico y microscópico, lográndose el mayor nivel de observación en el Microscopio Electrónico de Barrido

\begin{tabular}{|l|l|}
\hline & \multicolumn{1}{|c|}{ Observaciones } \\
\hline $\begin{array}{l}\text { Fijativo 1 } \\
\text { Pixoy macerado en } \\
\text { agua de cal }\end{array}$ & $\begin{array}{l}\text { Ésta es la única capa que es completamente mate, aun } \\
\text { observándola en el microscopio no se detectaron áreas con } \\
\text { brillo. }\end{array}$ \\
\hline $\begin{array}{l}\text { Fijativo 2 } \\
\text { Pixoy macerado en } \\
\text { agua + agua de cal 1:1 }\end{array}$ & $\begin{array}{l}\text { En este caso, en las zonas con concentraciones del fijativo, } \\
\text { generalmente en forma de escamas, se observó brillo, el cual a } \\
\text { nivel macroscópico es imperceptible. }\end{array}$ \\
\hline $\begin{array}{l}\text { Fijativo 3 } \\
\text { Pixoy macerado en } \\
\text { agua }\end{array}$ & $\begin{array}{l}\text { De las capas formadas por los fijativos, ésta es en la que se } \\
\text { observan, a nivel microscópico, mayor número de áreas con } \\
\text { brillo, las cuales corresponden a las zonas más concentradas. } \\
\text { En las observaciones macroscópicas se ve mate. }\end{array}$ \\
\hline
\end{tabular}

Tabla 3. Brillo de las capas formadas por los tres fijativos. 


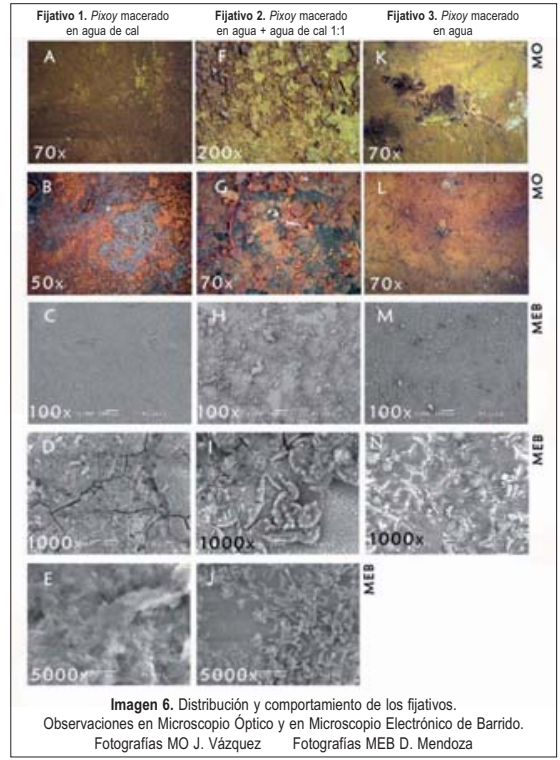

(MEB). En general ninguno de los tres fijativos forma una capa homogénea, en todos los casos se ven concentraciones diferenciales de material, todas las capas presentaron un patrón de craqueladuras (variando en cada caso). Esta prueba fue 
de gran relevancia, permitió entender por qué varía tanto el comportamiento entre los tres productos, así como entre las capas formadas por cada uno de ellos. (Imagen 6) (Tabla 4)

\begin{tabular}{|c|c|c|c|}
\hline & \multicolumn{3}{|c|}{ Observaciones } \\
\hline & Nivel macroscópico & Microscopio óptico & $\begin{array}{l}\text { Microscopio electrónico } \\
\text { de barrido }\end{array}$ \\
\hline $\begin{array}{l}\text { Fijativo } 1 \\
\text { Pixoy mace- } \\
\text { rado en agua } \\
\text { de cal }\end{array}$ & $\begin{array}{l}\text { La capa que se forma } \\
\text { es sumamente hete- } \\
\text { rogénea, en algunas } \\
\text { zonas se da mayor } \\
\text { concentración del fi- } \\
\text { jativo, lo que genera } \\
\text { manchas muy locali- } \\
\text { zadas. }\end{array}$ & $\begin{array}{l}\text { En las imágenes obtenidas se } \\
\text { ve claramente la capa gruesa } \\
\text { y de aspecto cristalino que se } \\
\text { forma con el fijativo } 1 \text {. Ade- } \\
\text { más es posible percibir múl- } \\
\text { tiples tonalidades en la capa } \\
\text { formada. Se puede apreciar } \\
\text { un patrón de craqueladuras } \\
\text { poligonales especificamen- } \\
\text { te en las zonas de mayor } \\
\text { concentración, áreas que } \\
\text { también son más oscuras. } \\
\text { Fotografias A y B. }\end{array}$ & $\begin{array}{l}\text { En este caso no se observa } \\
\text { una pelicula como tal, es una } \\
\text { capa gruesa, parcialmente } \\
\text { homogénea y craquelada. Es } \\
\text { de aspecto completamente } \\
\text { cristalino y en el mayor au- } \\
\text { mento se observa que la mez- } \\
\text { cla entre la cal y el mucilago } \\
\text { produjo un solo compuesto. } \\
\text { Fotografias C, D y E. }\end{array}$ \\
\hline $\begin{array}{l}\text { Fijativo } 2 \\
\text { Pixoy ma- } \\
\text { cerado en } \\
\text { agua + agua } \\
\text { de cal } 1: 1\end{array}$ & $\begin{array}{l}\text { La distribución de este } \\
\text { fijativo es mucho más } \\
\text { uniforme que el fijativo } \\
1 \text {, sin embargo no se } \\
\text { crea una capa homo- } \\
\text { génea. Las áreas más } \\
\text { oscuras corresponden } \\
\text { a manchas generadas } \\
\text { por la alta concentra- } \\
\text { ción del fijativo en su- } \\
\text { perficie. }\end{array}$ & $\begin{array}{l}\text { En el microscopio óptico se } \\
\text { observa claramente cómo } \\
\text { la capa se separa formando } \\
\text { "islas" entre las que quedan } \\
\text { espacios vacios, estas islas } \\
\text { corresponden a las cazoletas } \\
\text { o escamas. } \\
\text { Fotografias F y G. }\end{array}$ & $\begin{array}{l}\text { Se forma una capa comple- } \\
\text { tamente heterogénea en la } \\
\text { que se observa la separación } \\
\text { de los componentes, la parte } \\
\text { orgánica está escamada en } \\
\text { forma de "cazoletas" y la cal } \\
\text { forma pequeños cristales. Las } \\
\text { escamas son resultado de un } \\
\text { patrón de craqueladuras. } \\
\text { Fotografias } \mathrm{H}, \mathrm{I} \text { y. }\end{array}$ \\
\hline $\begin{array}{l}\text { Fijativo } 3 \\
\text { Pixoy ma- } \\
\text { cerado en } \\
\text { agua }\end{array}$ & $\begin{array}{l}\text { En este nivel de ob- } \\
\text { servación sus carac- } \\
\text { terísticas son muy } \\
\text { similares a la capa } \\
\text { generada por el fija- } \\
\text { tivo 2. La distribución } \\
\text { es heterogénea y las } \\
\text { zonas con mayor acu- } \\
\text { mulación se identifican } \\
\text { por ser más oscuras. }\end{array}$ & $\begin{array}{l}\text { Se observa una capa delgada } \\
\text { y homogénea, a diferencia de } \\
\text { las otras dos, a este nivel no } \\
\text { es posible identificar un pa- } \\
\text { trón de craqueladuras. Sus } \\
\text { características corresponden } \\
\text { más a una pelicula plástica. } \\
\text { Fotografias K y L. }\end{array}$ & $\begin{array}{l}\text { Este fijativo forma la capa me- } \\
\text { nos heterogénea, es mucho } \\
\text { más uniforme en su compo- } \\
\text { sición y distribución. Al mayor } \\
\text { aumento es posible identificar } \\
\text { un patrón de craqueladuras, } \\
\text { las cuales corresponden a los } \\
\text { "hilos" que se identificaron en } \\
\text { el microscopio petrográfico y } \\
\text { que se describe en el apar- } \\
\text { tado de caracterización del } \\
\text { pixoy. } \\
\text { Fotografias M y N. }\end{array}$ \\
\hline
\end{tabular}

Tabla 4. Distribución de los fijativos en superficie.

Consideraciones realizadas a distintos niveles de observación. 


\section{Nivel de impregnación y penetración}

Esta prueba determinó la cantidad de fijativo que logró penetrar e impregnar las partículas disgregadas de la capa pictórica. Para esta prueba se hicieron inclusiones en resina poliéster de los cortes estratigráficos de cada muestra, de esta forma fue posible observar en el microscopio óptico el comportamiento que tuvieron los productos. Cada fijativo tuvo un efecto distinto, siendo el fijativo 3 el que penetró e impregnó mejor la capa de color, mientras que el fijativo 1 produjo menor penetración e impregnación. (Imagen 7) (Tabla 5)

\begin{tabular}{|c|c|c|}
\hline & \multicolumn{2}{|c|}{ Observaciones } \\
\hline & Muestra original & Muestra problema \\
\hline $\begin{array}{l}\text { Fijativo } 1 \\
\text { Pixoy mace- } \\
\text { rado en agua } \\
\text { de cal }\end{array}$ & $\begin{array}{l}\text { No existe penetración o impregnación } \\
\text { del fijativo. La capa que forma el pro- } \\
\text { ducto es muy delgada, compacta y } \\
\text { opaca. Su distribución se adapta a la } \\
\text { textura de la superficie. }\end{array}$ & $\begin{array}{l}\text { Se observa una delgada y muy compacta } \\
\text { capa del fijativo sobre el estrato pictórico. } \\
\text { Ésta no penetra entre las particulas de } \\
\text { pigmento y es opaca. La impregnación } \\
\text { del producto es mínima y sumamente } \\
\text { superficial. En las zonas en las que el } \\
\text { estrato presenta depresiones hay mayor } \\
\text { concentración del fijativo. }\end{array}$ \\
\hline $\begin{array}{l}\text { Fijativo } 2 \\
\text { Pixoy mace- } \\
\text { rado en agua } \\
+ \text { agua de } \\
\text { cal } 1: 1\end{array}$ & $\begin{array}{l}\text { En las muestras originales se ob- } \\
\text { serva una capa mucho más delgada } \\
\text { que en las problema. La distribución } \\
\text { es completamente heterogénea, de } \\
\text { hecho existen zonas donde no se } \\
\text { observa la presencia del fijativo. No } \\
\text { hay penetración ni impregnación del } \\
\text { producto. }\end{array}$ & $\begin{array}{l}\text { La capa que forma es muy gruesa y traslú- } \\
\text { cida, respecto al fijativo } 1 \text {. Su aspecto es } \\
\text { similar a una veladura. Su distribución } \\
\text { es regular y se adapta a la superficie de la } \\
\text { muestra. Se observa mayor penetración } \\
\text { e impregnación que con el fijativo 1, en } \\
\text { algunas zonas se puede ver cómo rodea } \\
\text { algunas partículas de pigmento. }\end{array}$ \\
\hline $\begin{array}{l}\text { Fijativo } 3 \\
\text { Pixoy mace- } \\
\text { rado en agua }\end{array}$ & $\begin{array}{l}\text { En esta muestra se observó una dis- } \\
\text { tribución bastante uniforme en la su- } \\
\text { perficie, el fijativo se adaptó a la tex- } \\
\text { tura de la superficie de la muestra y } \\
\text { deja una capa delgada, opaca y más } \\
\text { compacta que el fijativo } 2 \text { pero menos } \\
\text { que el fijativo } 3 \text {. No hay penetración ni } \\
\text { impregnación del fijativo. }\end{array}$ & $\begin{array}{l}\text { Sobre la superficie de la muestra se ob- } \\
\text { serva una capa gruesa, menos traslúcida } \\
\text { que la del fijativo } 2 \text { y un poco más com- } \\
\text { pacta. Se distribuye de forma homogénea } \\
\text { sobre la muestra. Se observa cierto nivel } \\
\text { de penetración e impregnación en la capa } \\
\text { de color, es muy superficial, sólo en zo- } \\
\text { nas muy localizadas se puede ver que el } \\
\text { fijativo casi penetra hasta la superficie del } \\
\text { enlucido fino. }\end{array}$ \\
\hline
\end{tabular}

Tabla 5. Nivel de impregnación y penetración de los diversos fijativos en muestras originales y muestras problema. 


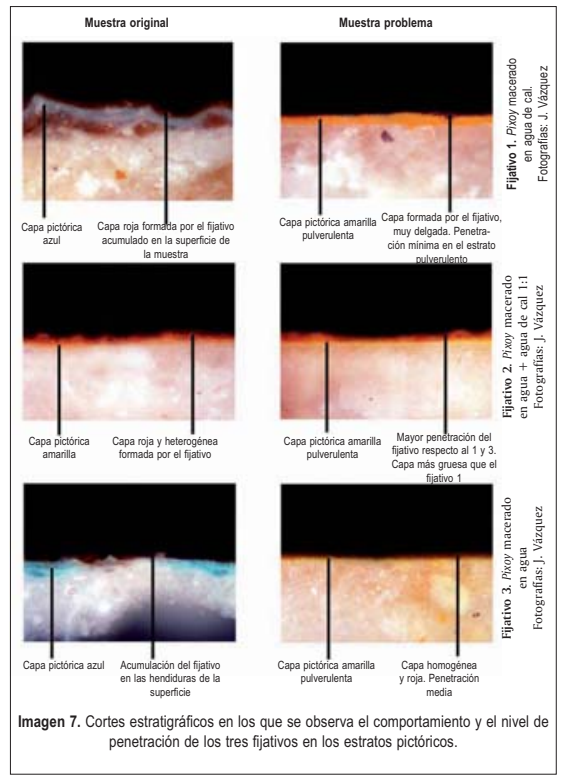




\section{Resistencia a la abrasión}

Esta prueba consistió en pasar suavemente el dedo sobre la capa de color de las muestras problema, y así observar si continuaba el problema de pulverulencia. En todos los casos el color se desprendió, variando la cantidad de pigmento esparcido por la abrasión de acuerdo con cada uno de los fijativos. Las muestras en las que se empleó el fijativo 2 fueron las que presentaron un mayor nivel de pulverulencia, mientras que las del fijativo 1 mostraron menor cantidad de pigmento suelto, quedando en un nivel intermedio el fijativo 3 (Ruiz, 2007: 140).

\section{Adhesión de los pigmentos al soporte}

Con el fin de conocer la adhesividad que ofrecieron los fijativos a las partículas disgregadas, se hizo una sencilla prueba que consistió en adherir una cinta adhesiva a la superficie de las muestras y después desprenderla. Esto se hizo de dos formas, una con todo el adhesivo y otra en la cual se eliminó parcialmente (adhiriéndola a una tela antes de utilizarla en las muestras). En todos los casos se observó el desprendimiento de una cantidad considerable del estrato de color. (Tabla 6)

PRUEBAS MECÁNICAS. ANẢLISIS DE RESULTADOS

La falta de impregnación del fijativo y de penetración en el estrato disgregado se debió al uso de agua como solvente y a que el extracto de pixoy es un polisacárido. El agua, por ser un líquido polar, presenta alta tensión superficial, lo que disminuye su capacidad de penetración y, al mezclarse con la goma, la cual

\begin{tabular}{|l|l|}
\hline & \multicolumn{1}{|c|}{ Observaciones } \\
\hline $\begin{array}{l}\text { Fijativo 1 } \\
\begin{array}{l}\text { Pixoy macerado } \\
\text { en agua de cal }\end{array}\end{array}$ & $\begin{array}{l}\text { Se desprende una pequeña cantidad de partículas de color. Respec- } \\
\text { to a los otros fijativos este caso es en el que existe menor despren- } \\
\text { dimiento. También se observa parte del fijativo adherido a la cinta. } \\
\text { Sobre el enlucido se conservó color adherido a su superficie. }\end{array}$ \\
\hline $\begin{array}{l}\text { Fijativo 2 Pixoy } \\
\text { macerado en } \\
\text { agua + agua de } \\
\text { cal 1:1 }\end{array}$ & $\begin{array}{l}\text { En este caso el desprendimiento de color es muy alto, es en el que } \\
\text { se observó mayor cantidad de color en la cinta. En algunas zonas de } \\
\text { la mente expuesto. También se desprende el fijativo. }\end{array}$ \\
\hline $\begin{array}{l}\text { Fijativo 3 } \\
\text { Pixoy macerado completa- } \\
\text { en agua }\end{array}$ & $\begin{array}{l}\text { El nivel de desprendimiento es intermedio entre el fijativo 1 y 2. Se } \\
\text { observa una pérdida superficial de los pigmentos, el color no se per- } \\
\text { dió hasta el nivel del enlucido. }\end{array}$ \\
\hline
\end{tabular}

Tabla 6. Adhesión de los pigmentos al soporte. Resultados de la prueba para cada fijativo. 
está conformada por polisacáridos de alto peso molecular, conformaron una solución densa y viscosa (Ruiz, 2007: 144). A mayor viscosidad, menor capacidad del líquido a difundirse sobre y entre un sólido, por lo tanto menor penetración e impregnación (Newey, et al., 1984: 18).

La principal razón de la heterogeneidad de las capas formadas por los fijativos fue el sistema de aplicación. Las condiciones debieron ser más controladas, por ejemplo buscar la horizontalidad total de todas las muestras para evitar zonas con mayor concentración de fijativo y emplear un sistema de asperjado más preciso. Es muy importante considerar que una distribución heterogénea tiene como consecuencia, además de una alteración visual al producir manchas más oscuras en las zonas más concentradas, un trabajo estructural irregular y por lo tanto inestable (Ruiz, 2007: 144).

Al evaluar las características de la superficie creada por cada uno de los fijativos fueron muy evidentes las diferencias que existieron entre cada producto y se concluyó que la principal causa de esta situación fue la forma en que se prepararon y obtuvieron los consolidantes, siendo determinante el uso de agua de cal o bien de agua potable en la maceración de la corteza.

El trabajo y la distribución heterogénea de los tres fijativos junto con la falta de penetración e impregnación de las partículas pulverulentas impidieron devolver al estrato de color la adhesión y la cohesión necesaria para estabilizarla. El hecho de que los productos no lograran penetrar por completo hasta llegar al enlucido de cal impidió que se formara la interfase necesaria para unir la capa pictórica disgregada a su soporte, por lo que el roce ligero del dedo sobre la superficie de las muestras ocasionó el desprendimiento del pigmento (Ruiz, 2007: 146).

PRUEBAS DE SOLUBILIDAD

Para probar la solubilidad de los tres fijativos a los seis meses de haberlos aplicado, se emplearon diversos disolventes comúnmente utilizados en conservación, algunos polares y otros no polares. La prueba se hizo rodando sobre la superficie de las muestras hisopos de bambú con algodón impregnados con los disolventes, Los resultados obtenidos de esta prueba indicaron que la mayor solubilidad se presenta con los solventes polares, quedando en el siguiente orden: agua, aguaalcohol 1:1, alcohol, acetona, saliva, ${ }^{4}$ xilol y thinner (Ruiz, 2007:140-142).

SOLUBILIDAD. ANÄLISIS DE RESULTADOS

Las pruebas de solubilidad demostraron que los solventes polares son los más efectivos en la disolución de los fijativos de pixoy, a diferencia del xilol y el thinner que son solventes totalmente no polares. Estos resultados eran esperados, ya

\footnotetext{
${ }^{4}$ En restauración es muy común el empleo de saliva para realizar procesos de limpieza. Al contener agua y una gran cantidad de enzimas, facilita la solubilidad de suciedad y en algunos casos de capas de protección y barnices deteriorados.
} 
que los tres fijativos son sustancias polares y por lo tanto de mayor solubilidad con disolventes que tienen una polaridad igual o muy similar a ellos. Con esto se advierte que los productos elaborados con el pixoy se mantienen solubles después de seis meses de haberse aplicado, lo cual indica que por lo menos en ese periodo el mucílago no ha sufrido un deterioro por reticulación y/o despolimerización que disminuya su solubilidad y altere sus propiedades físico-mecánicas. Aspecto que debido a la ineficacia de los productos probados como fijativos pierde relevancia (Ruiz, 2007:146).

\section{Conclusiones}

Las observaciones hechas en campo, antes de esta investigación, indicaban que el producto elaborado con pixoy sí resultaba efectivo como fijativo, y en algunos casos como consolidante de estuco disgregado. Sin embargo, es importante considerar que durante las breves temporadas del trabajo de campo, por falta de tiempo y herramientas, no había sido posible evaluar a profundidad si la disminución de la disgregación y pulverulencia de los materiales era durable y completamente efectiva. Ahora, gracias a las pruebas y observaciones hechas en esta evaluación es posible aseverar que los productos preparados con el extracto de pixoy, siguiendo la metodología expuesta, no son efectivos.

Se considera que la metodología de evaluación empleada fue positiva y suficiente para esta investigación, pero es posible mejorar algunos aspectos y considerar más pruebas, como por ejemplo contemplar el comportamiento de los productos ante agentes biológicos como algas, líquenes, bacterias, hongos, entre otros, en particular si el producto a evaluar se va a utilizar en contextos climáticos cálidos y húmedos como en la zona maya.

Más allá de la ineficacia observada en los productos elaborados con el extracto de pixoy como fijativos, fue de gran relevancia para esta investigación el retomar y evaluar un producto que se mantuvo en uso por varios años sin saber si realmente estaba cumpliendo su función.

Es fundamental insistir en el hecho de que mientras no se conozca a profundidad, un producto y sus efectos, éste no debe ser empleado, bajo ninguna circunstancia y sin importar su naturaleza, en bienes culturales que son únicos, irrepetibles e irremplazables.

Hasta el momento no ha sido posible encontrar un producto realmente efectivo en el control del efecto de pulverulencia de capas pictóricas de pintura mural y relieves policromos en sitios del área maya. Además del uso del agua de cal y de los extractos vegetales, se han realizado intervenciones con nanopartículas de hidróxido de calcio, producto elaborado en la Universidad de Florencia por el equipo de investigación del Dr. Piero Baglioni, producto que, a pesar de resultar efectivo hasta el momento, sigue en evaluación y experimentación (Baglioni et al., 1997; Giorgi et al., 2000; Ambrosi et al., 2001; Baglioni et al., 2006). 
Es importante enfatizar que, además de la necesidad que hay de encontrar fijativos efectivos para los bienes estudiados, hay mucho más por trabajar en el sentido de la conservación integral. A partir de la experiencia obtenida a través de esta investigación junto con el trabajo profesional en campo, se ha comprobado que es fundamental conocer todas las causas de deterioro que rodean la pintura mural y los relieves a intervenir. Consolidar una capa pictórica no va a funcionar más de dos semanas si el muro tiene serios problemas de humedad y de sales; o si en el techo existen filtraciones de agua por pérdida de material de rejunteo; o bien, si hay una acumulación considerable de hojas y plantas en la parte posterior del muro que da soporte a la pintura o al relieve. Todos estos aspectos identificados a partir de un diagnóstico general deberán ser solucionados, ya que es la única manera de garantizar la conservación integral del bien por más tiempo, para lo cual es indispensable que diversos profesionales como restauradores, arqueólogos y arquitectos trabajen de forma integral y conjunta aportando opiniones y soluciones a los problemas que se enfrentan en la conservación de los sitios arqueológicos (Ruiz, 2007: 154).

\section{Bibliografía}

Alonso, Alejandra y Patricia Meehan

2001 "Primer informe de los trabajos de conservación y restauración de los elementos decorativos de la Acrópolis de la zona arqueológica de Ek 'Balam, Yucatán. Primera temporada". Informe técnico. 95 pp. México: INAH-CNCPC Subdirección de Conservación Arqueológica y Acabados Arquitectónicos. Archivo de la CNCPC.

Alonso, Alejandra, Patricia Meehan, Yareli Jáidar, Octavio Murillo y Cristina Ruiz

2002 "Segundo informe de los trabajos de conservación y restauración de los elementos decorativos de la Acrópolis de la zona arqueológica de Ek 'Balam, Yucatán. Segunda temporada". Informe técnico. 58 pp. México: INAH-CNCPC Subdirección de Conservación Arqueológica y Acabados Arquitectónicos. Archivo de la CNCPC.

Alonso, Alejandra, Patricia Meehan, Yareli Jáidar y Cristina Ruiz

2003 "Tercer informe de los trabajos de conservación y restauración de los elementos decorativos de la Acrópolis de la zona arqueológica de Ek 'Balam, Yucatán. Tercera temporada". Informe técnico. $60 \mathrm{pp}$. México: INAH-CNCPC Subdirección de Conservación Arqueológica y Acabados Arquitectónicos. Archivo de la CNCPC.

Alonso, Alejandra, Demetrio Mendoza, Manuel Espinosa, José Luis Ruvalcaba, Peter Vandenabeele, Javier Vázquez y Cristina Ruiz

2005 "Resultados preliminares sobre la caracterización de materiales constitutivos y tecnología de la pintura mural de la Acrópolis de la zona arqueológica de Ek 'Balam, Yucatán”, La ciencia de materiales y su impacto en la arqueología. 
Vol. II, pp. 191-217. Demetrio Mendoza, Jesús Arenas y Ventura Rodríguez (eds). México: Academia Mexicana de Ciencia de Materiales A. C. Innovación Editorial Lagares México.

Ambrosi, Mora, Luigi Dei, Rodorico Giorgi, Chiara Neto y Piero Baglioni

2001 "Colloidal particles of $\mathrm{Ca}(\mathrm{OH}) 2$ : Properties and applications to restoration of frescoes", Langmuir 17, pp. 4251-4255.

Baglioni, P., L.. Dei, F. Piqué, G. Sarti y E. Ferroni

1997 "New Autogenous Lime-Based Grouths Used in the Conservation of LimeBased Wall Paintings", Studies in Conservation No. 42, pp. 43-54.

Baglioni, Piero, Ramón Carrasco, David Chelazzi, Marinés Colón, Alice Desprat y Rodorico Giorgi

2006 "The Maya Site of Calakmul: In Situ Preservation of Wall Paintings and Limestones using Nanotechnologies", The Object in Context. IIC Munich Congress, pp. 162-169.

Brajer, Isabelle y Nicole Kalsbeek

1999 "Limewater Absorption and Calcite Crystal Formation on a Limewater-Impregnated Secco Wall Painting", Studies in Conservation No. 44, pp. 145156.

Budavari, Susan (ed.)

1989 The Merk Index. 11th edition Centennial edition. 1606 pp., New Jersey: Ed. Merck and Co.

Castro, Ma. del Carmen y Martha Tapia

1993 "Intervenciones anteriores de conservación: seguimiento y evaluación". Tesis de licenciatura. 299 pp. México: INAH-ENCrM.

Cedillo, Luciano

1991 "La conservación de zonas arqueológicas: tres décadas de trabajo". Tesis de licenciatura. 371 pp. México: INAH-ENCRM.

Cedillo, Luciano

1993 "Estuco: Informe de los métodos desarrollados en México", Conservación arqueológica in situ. Henry Hodges (ed.). Actas de la reunión 6-13 abril 1986 en México. INAH GCI. EUA. pp. 96-103.

Cedillo, Luciano, Gabriela García Lascurain, Luz de Lourdes Herbert

1997 "Trabajos de conservación en zonas arqueológicas del área maya", Temas y problemas. 1er coloquio del Seminario de Estudio del Patrimonio Artístico. Conservación, restauración y defensa, pp. 125-136, México: unam-1IE.

Chan, Julio

1991 Conservación de la zona arqueológica de Balamkú, Campeche, Marzo 1991. Informe técnico. s/p. México: INAH-CNCPC. Archivo de la CNCPC.

RUIZ MARTÍN / EL PIXOY COMO MATERIAL DE CONSERVACIÓN PARA PINTURA MURAL 
García, Valeria y Renata Schneider

1994 "Informe de las intervenciones realizadas sobre el relieve en estuco de la subestructura 1 " $\mathrm{A}$ " de la plaza 2 de Balamkú, Campeche", Informe técnico. 53 pp. México: CNRPC-ENCRM-INAH. Archivo de la CNCPC.

Giorgi, Rodorico, Luigi Dei y Piero Baglioni

$2000 \quad$ "A new method for consolidating wall paintings based on dispersions of lime in alcohol", Studies in Conservation No. 45, pp. 154-161.

Horie, C.V.

1990 Materials for Conservation. Organic Consolidants, Adhesives and Coatings, 281 pp. Londres: Butterworths.

Jáidar, Yareli 2006

"Los extractos vegetales usados como aditivos en los morteros de cal con fines de conservación". Tesis de licenciatura. 314 pp. México: INAH-ENCrM.

Jáidar, Yareli y Cristina Ruiz

2004 Cuarto informe de los trabajos de conservación y restauración de los elementos decorativos de la Acrópolis de la zona arqueológica de Ek 'Balam, Yucatán. Cuarta temporada. Informe técnico. 75 pp. México: INAH-CNCPC Subdirección de Conservación Arqueológica y Acabados Arquitectónicos. Archivo de la CNCPC.

Jáidar, Yareli, Cristina Ruiz y Demetrio Mendoza (en prensa)

2006 "Un acercamiento a la caracterización de extractos vegetales empleados en conservación", Archaeological and Art Issues in Materials Science. Academia Mexicana de Ciencia de Materiales, A.C. México.

Littmann, Edwin

1960 "Ancient Mesoamerican Mortars, Plasters and Stuccos: the use of Bark Extracts in Lime Plasters", American Antiquity Vol. 25 No. 4 pp. 593-597 Salt Lake City: The Society for American Archaeology University of Utah Press.

1965 "The Classification and Analysis of Ancient Calcareous Materials", American Antiquity Vol. 31 No. 6 pp. 875-878 Salt Lake City: The Society for American Archaeology University of Utah Press.

Magaloni, Diana

1997 "La cal y los mayas: un ejemplo de la necesidad de contextualizar la investigación sobre el México prehispánico", La pintura mural prehispánica en México. Boletín informativo Año III No. 6-7. Leticia Staines (ed.) pp. 5-8, México: UNAM-IIE.

2000 "El arte en el hacer: técnica pictórica y color en las pinturas de Bonampak", La pintura Mural Prehispánica en México. Área maya. Bonampak. Tomo II. pp. 49-78, Beatriz de la Fuente (coord.). México: unam-IIE.

2001 "Materiales y técnicas de la pintura mural maya", La pintura Mural Prehispánica en México II. Área maya. Tomo III. pp. 155-198, Leticia Staines (coord.) México: UNam-IIE. 
Martínez de las Marías, $\mathrm{P}$.

1972 Química y fisica de los altos polímeros y materias plásticas. 296 pp. Madrid: Alhambra.

Matteini, Mauro y Moles Arcangelo (trad. Carolusa González)

1989 La Chimica nel restauro: I materiali dell arte pittorica. 18 pp. Firenze.

Matteini, Mauro y Moles Arcanuelo

2001 Ciencia y restauración. Método de investigación. 304 pp. Andalucía: Junta de Andalucía Consejería de Cultura ı.P.

Mateos, Frida,

1997 Toniná: la pintura mural y los relieves en estuco. Técnica de manufactura. 104 pp. México: Colección científica Serie Arqueología ıмн. Centro de Investigaciones Humanísticas de Mesoamérica y el estado de Chiapas UNAM.

Masschelein, Liliane (trad. Jane Bridgland, Sue Walston y A. E. Werner)

1995 Ancient Binding Media, Varnishes and Adhesives. pp. 1-51 Roma: Iccrom.

Mora, Paulo y Laura Mora,

1984 Conservation of Wall Painting. 494 pp. Londres: Butterworths ıccrom.

Newey, Charles, Ruth Boff, Vincent Daniels, Michael Pascoe y Norman Tennent

1987 Science for conservators. Adhesives and Coatings. Book 3. Conservation Science Teaching Series. 135 pp. Londres: The Conservation Unit.

Pennington, P.D. y J. Sarukhan

1998 Árboles tropicales de México. Manual para la identificación de las principales especies. 382 pp. México: UNAM-Fondo de Cultura Económica-Instituto de Biología.

Roys, Ralph

1931 The Etnobotany of the Maya. p. 276. Nueva Orleans: Department of Middle American Research. Tulane University.

Ruiz, Cristina

2007 "El uso del pixoy como fijativo de capas pictóricas pulverulentas al seco en pintura mural prehispánica: El caso de la Estructura 1 de Ek'Balam, Yucatán". Tesis de licenciatura. México: INAH-ENCRM, $212 \mathrm{pp}$.

Standley, Paul

1977 "La Flora Yucatanense" Enciclopedia Yucatanense. Capítulo I Generalidades. p. 273. México: Edición oficial del Gobierno de Yucatán, 2aㅡ edición, Ciudad de México

Thompson, Edward A.

1965 People of the Serpent. Life and Adventure Among the Mayas. 301 pp. Nueva York: Capricorn Books.

RUIZ MARTÍN / EL PIXOY COMO MATERIAL DE CONSERVACIÓN PARA PINTURA MURAL 


\section{Vázquez de Ágredos, María Luisa}

2004 "Gomas, resinas, y aceites: sus propiedades, preparación y uso en la pintura mural maya". Ponencia presentada en el VI Congreso Internacional de Mayistas. Villahermosa, Tabasco. Julio de 2004. Texto inédito copia del autor.

2006 "Recursos materiales y técnicas pictóricas en los murales de las Tierras Bajas Mayas". Tesis de doctorado. 1193 pp. Valencia: Universidad de Valencia.

Vázquez, Ximena y Alejandra M. Villegas

1996 "Los estucos modelados del Palacio y Templo de las Inscripciones de Palenque: una metodología de análisis para la técnica de manufactura". Tesis de Licenciatura. 126 pp. México: INAH-ENCRM.

Vázquez, Javier y Rodrigo Velásquez (mecanoescrito)

1994 Estudio del estado de conservación en materiales constitutivos de relieves en estuco, mortero y pintura mural de la zona arqueológica de Palenque, Chiapas, a través del análisis de sus características morfológicas, microestructurales y de composición química. Texto inédito copia del autor. 185 pp. 